\title{
A note on complex-hyperbolic Kleinian groups
}

\author{
Subhadip Dey* $\quad$ Michael Kapovich ${ }^{\dagger}$
}

May 2, 2020

\begin{abstract}
Let $\Gamma$ be a discrete group of isometries acting on the complex hyperbolic $n$-space $\mathbb{H}_{\mathbb{C}}^{n}$. In this note, we prove that if $\Gamma$ is convex-cocompact, torsion-free, and the critical exponent $\delta(\Gamma)$ is strictly lesser than 2 , then the complex manifold $\mathbb{H}_{\mathbb{C}}^{n} / \Gamma$ is Stein. We also discuss several related conjectures.
\end{abstract}

The theory of complex hyperbolic manifolds and complex-hyperbolic Kleinian groups (i.e. discrete holomorphic isometry groups of complex hyperbolic spaces $\mathbb{H}_{\mathbb{C}}^{n}$ ) is a rich mixture of Riemannian and complex geometry, topology, dynamics, symplectic geometry and complex analysis. The purpose of this note is to discuss interactions of the theory of complex-hyperbolic Kleinian groups and the function theory of complex-hyperbolic manifolds. Let $\Gamma$ be a discrete group of isometries acting on the complex-hyperbolic $n$-space, $\mathbb{H}_{\mathbb{C}}^{n}$, the unit ball $\mathbf{B}^{n} \subset \mathbb{C}^{n}$ equipped with the Bergmann metric. A fundamental numerical invariant associated with $\Gamma$ is the critical exponent $\delta(\Gamma)$ of $\Gamma$, defined by

$$
\delta(\Gamma)=\inf \left\{s: \sum_{\gamma \in \Gamma} e^{-s \cdot d(x, \gamma x)}<\infty\right\}
$$

where $x \in \mathbb{H}_{\mathbb{C}}^{n}$ is any ${ }^{1}$ point. The critical exponent measures the rate of exponential growth the $\Gamma$-orbit $\Gamma x \subset \mathbb{H}_{\mathbb{C}}^{n}$; it also equals the Haussdorff dimension of the conical limit set of $\Gamma$, see $[7]$ and [8].

Our main result is:

Theorem 1. Suppose that $\Gamma<\operatorname{Aut}\left(\mathbf{B}^{n}\right)$ is a convex-cocompact, torsion-free discrete subgroup satisfying $\delta(\Gamma)<2$. Then $M_{\Gamma}=\mathbf{B}^{n} / \Gamma$ is Stein.

\footnotetext{
${ }^{*}$ Dept. of Math., UC Davis, One Shields Ave, Davis, CA 95616, email: sdey@math.ucdavis.edu

${ }^{\dagger}$ Dept. of Math., UC Davis, One Shields Ave, Davis, CA 95616, email: kapovich@math.ucdavis.edu

${ }^{1} \delta(\Gamma)$ does not depend on the choice of $x \in \mathbb{H}_{\mathbb{C}}^{n}$.
} 
The condition on the critical exponent in the above theorem is sharp since, for a complex Fuchsian subgroup $\Gamma<\operatorname{Aut}\left(\mathbf{B}^{n}\right), \delta(\Gamma)=2$, but the quotient $M_{\Gamma}=\mathbf{B}^{n} / \Gamma$ is non-Stein because the convex core of $M_{\Gamma}$ is a complex curve, see Example 4. On the other hand, if $\Gamma$ is a torsion-free real Fuchsian subgroup or a small deformation of such (see Example 3), then $\Gamma$ satisfies the condition of the above theorem.

The main ingredients in the proof of Theorem 1 are Proposition 11 and Theorem 15. The condition "convex-cocompact" is only used in Proposition 11, whereas Theorem 15 holds for any torsion-free discrete subgroup $\Gamma<\operatorname{Aut}\left(\mathbf{B}^{n}\right)$ satisfying $\delta(\Gamma)<2$.

Conjecture 2. Theorem 1 holds if we omit the "convex-cocompact" assumption on $\Gamma$.

In section 4 we discuss other conjectural generalizations of Theorem 1 and supporting results.

Acknowledgement. The second author was partly supported by the NSF grant DMS16-04241.

\section{Preliminaries}

In this section, we recall some definitions and basic facts about the $n$-dimensional complex hyperbolic space, we refer to $[9,11]$ for details.

Consider the $n$-dimensional complex vector space $\mathbb{C}^{n+1}$ equipped with the pseudohermitian bilinear form

$$
\langle z, w\rangle=-z_{0} \bar{w}_{0}+\sum_{k=1}^{n} z_{k} \bar{w}_{k}
$$

and define the quadratic form $q(z)$ of signature $(n, 1)$ by $q(z):=\langle z, z\rangle$. Then $q$ defines the negative light cone $V_{-}:=\{z: q(z)<0\} \subset \mathbb{C}^{n+1}$. The projection of $V_{-}$in the projectivization of $\mathbb{C}^{n+1}, \mathbb{P}^{n}$, is an open ball which we denote by $\mathbf{B}^{n}$.

The tangent space $T_{[z]} \mathbb{P}^{n}$ is naturally identified with $z^{\perp}$, the orthogonal complement of $\mathbb{C} z$ in $V$, taken with respect to $\langle\cdot, \cdot\rangle$. If $z \in V_{-}$, then the restriction of $q$ to $z^{\perp}$ is positivedefinite, hence, $\langle\cdot, \cdot\rangle$ project to a hermitian metric $h$ (also denoted $\langle\cdot, \cdot\rangle_{h}$ ) on $\mathbf{B}^{n}$. The complex hyperbolic $n$-space $\mathbb{H}_{\mathbb{C}}^{n}$ is $\mathbf{B}^{n}$ equipped with the hermitian metric $h$. The boundary $\partial \mathbf{B}^{n}$ of $\mathbf{B}^{n}$ in $\mathbb{P}^{n}$ gives a natural compactification of $\mathbf{B}^{n}$.

In this note, we usually denote the complex hyperbolic $n$-space by $\mathbf{B}^{n}$. The real part of the hermitian metric $h$ defines a Riemannian metric $g$ on $\mathbf{B}^{n}$. The sectional curvature of $g$ varies between -4 and -1 . We denote the distance function on $\mathbf{B}^{n}$ by $d$. The distance function satisfies

$$
\cosh ^{2}(d(0, z))=\left(1-|z|^{2}\right)^{-1} .
$$

A real linear subspace $W \subset \mathbb{C}^{n+1}$ is said to be totally real with respect to the form (1) if for any two vectors $z, w \in W,\langle z, w\rangle \in \mathbb{R}$. Such a subspace is automatically totally real in the usual sense: $J W \cap W=\{0\}$, where $J$ is the almost complex structure on $V$. (Real) 
geodesics in $\mathbf{B}^{n}$ are projections of totally real indefinite (with respect to $q$ ) 2-planes in $\mathbb{C}^{n+1}$ (intersected with $V_{-}$). For instance, geodesics through the origin $0 \in \mathbf{B}^{n}$ are Euclidean line segments in $\mathbf{B}^{n}$. More generally, totally-geodesic real subspaces in $\mathbf{B}^{n}$ are projections of totally real indefinite subspaces in $\mathbb{C}^{n+1}$ (intersected with $V_{-}$). They are isometric to the real hyperbolic space $\mathbb{H}_{\mathbb{R}}^{n}$ of constant sectional curvature -1 .

Complex geodesics in $\mathbf{B}^{n}$ are projections of indefinite complex 2-planes. Complex geodesics are isometric to the unit disk with the hermitian metric

$$
\frac{d z d \bar{z}}{\left(1-|z|^{2}\right)^{2}}
$$

which has constant sectional curvature -4 . More generally, $k$-dimensional complex hyperbolic subspaces $\mathbb{H}_{\mathbb{C}}^{k}$ in $\mathbf{B}^{n}$ are projections of indefinite complex $(k+1)$-dimensional subspaces (intersected with $V_{-}$).

All complete totally-geodesic submanifolds in $\mathbb{H}_{\mathbb{C}}^{n}$ are either real or complex hyperbolic subspaces.

The group $\mathrm{U}(n, 1) \cong \mathrm{U}(q)$ of (complex) automorphisms of the form $q$ projects to the group $\operatorname{Aut}\left(\mathbf{B}^{n}\right) \cong \mathrm{PU}(n, 1)$ of complex (biholomorphic, isometric) automorphisms of $\mathbf{B}^{n}$. The group $\operatorname{Aut}\left(\mathbf{B}^{n}\right)$ is linear, its matrix representation is given, for instance, by the adjoint representation, which is faithful since $\operatorname{Aut}\left(\mathbf{B}^{n}\right)$ has trivial center.

A discrete subgroup $\Gamma$ of $\operatorname{Aut}\left(\mathbf{B}^{n}\right)$ is called a complex-hyperbolic Kleinian group. The accumulation set of an $(\mathrm{y})$ orbit $\Gamma x$ in $\partial \mathbf{B}^{n}$ is called the limit set of $\Gamma$ and denoted by $\Lambda(\Gamma)$. The complement of $\Lambda(\Gamma)$ in $\partial \mathbf{B}^{n}$ is called the domain of discontinuity of $\Gamma$ and denoted by $\Omega(\Gamma)$. The group $\Gamma$ acts properly discontinuously on $\mathbf{B}^{n} \cup \Omega(\Gamma)$.

For a (torsion-free) complex-hyperbolic Kleinian group $\Gamma$, the quotient $\mathbf{B}^{n} / \Gamma$ is a Riemannian orbifold (manifold) equipped with push-forward of the Riemannian metric of $\mathbf{B}^{n}$. We reserve the notation $M_{\Gamma}$ to denote this quotient. The convex core of $M_{\Gamma}$ is the the projection of the closed convex hull of $\Lambda(\Gamma)$ in $\mathbf{B}^{n}$. The subgroup $\Gamma$ is called convexcocompact if the convex core of $M_{\Gamma}$ is a nonempty compact subset. Equivalently (see [3]), $\bar{M}_{\Gamma}=\left(\mathbf{B}^{n} \cup \Omega(\Gamma)\right) / \Gamma$ is compact.

Below are two interesting examples of convex-cocompact complex-hyperbolic Kleinian groups which will also serve as illustrations our results.

Example 3 (Real Fuchsian subgroups). Let $\mathbb{H}_{\mathbb{R}}^{2} \subset \mathbf{B}^{n}$ be a totally real hyperbolic plane. This inclusion is induced by an embedding $\rho: \operatorname{Isom}\left(\mathbb{H}_{\mathbb{R}}^{2}\right)=\operatorname{PSL}(2, \mathbb{R}) \rightarrow \operatorname{Aut}\left(\mathbf{B}^{n}\right)$ whose image preserves $\mathbb{H}_{\mathbb{R}}^{2}$. Let $\Gamma^{\prime}<\operatorname{Isom}\left(\mathbb{H}_{\mathbb{R}}^{2}\right)$ be a uniform lattice. Then $\Gamma=\rho\left(\Gamma^{\prime}\right)$ preserves $\mathbb{H}_{\mathbb{R}}^{2}$ and acts on it cocompactly. Such subgroups $\Gamma<\operatorname{Aut}\left(\mathbf{B}^{n}\right)$ will be called real Fuchsian subgroups. The compact surface-orbifold $\Sigma=\mathbb{H}_{\mathbb{R}}^{2} / \Gamma$ is the convex core of $M_{\Gamma}$. The critical exponent $\delta(\Gamma)$ is 1 .

Let $\Gamma_{t}, t \geq 0$, be a continuous family of deformations of $\Gamma_{0}=\Gamma$ in $\operatorname{Aut}\left(\mathbf{B}^{n}\right)$ such that $\Gamma_{t}$ 's, for $t>0$, are convex-cocompact but not real Fuchsian. Such deformation exist as long as $\Gamma_{t}$ is, say, torsion-free, see e.g. [15]. The groups $\Gamma_{t}, t>0$, are called real quasi-Fuchsian subgroups. The critical exponents of such subgroups are strictly greater than 1 . 
Example 4 (Complex Fuchsian subgroups). Let $\Gamma^{\prime}$ be a cocompact subgroup of $\mathrm{SU}(1,1)$, the identity component isometry group of the real-hyperbolic plane (modulo $\mathbb{Z}_{2}$ ) and let $\mathrm{SU}(1,1) \rightarrow \mathrm{SU}(n, 1)$ be any embedding. Note that $\mathrm{SU}(n, 1)$ modulo center (isomorphic to $\left.\mathbb{Z}_{n+1}\right)$ is isomorphic to $\mathrm{PU}(n, 1)$. By taking compositions, we get a representation $\rho: \Gamma^{\prime} \rightarrow \mathrm{PU}(n, 1)$. Then $\Gamma:=\rho\left(\Gamma^{\prime}\right)$ leaves a complex geodesic invariant in $\mathbf{B}^{n}$. Such subgroups $\Gamma$ will be called complex Fuchsian subgroups. In this case, $\operatorname{core}\left(M_{\Gamma}\right)=\mathbb{H}_{\mathbb{C}}^{1} / \Gamma$ is a compact complex curve in $M_{\Gamma}$ where $\mathbb{H}_{\mathbb{C}}^{1}$ is the $\Gamma$-invariant complex geodesic. The critical exponent $\delta(\Gamma)$ is 2 .

\section{Generalities on complex manifolds}

By a complex manifold with boundary $M$, we mean a smooth manifold with (possibly empty) boundary $\partial M$ such that $\operatorname{int}(M)$ is equipped with a complex structure and that there exists a smooth embedding $f: M \rightarrow X$ to an equidimensional complex manifold $X$, biholomorphic on $\operatorname{int}(M)$. A holomorphic function on $M$ is a smooth function which admits a holomorphic extension to a neighborhood of $M$ in $X$.

Let $X$ be a complex manifold and $Y \subset X$ is a codimension 0 smooth submanifold with boundary in $X$. The submanifold $Y$ is said to be strictly Levi-convex if every boundary point of $Y$ admits a neighborhood $U$ in $X$ such that the submanifold with boundary $Y \cap U$ can be written as

$$
\{\phi \leq 0\},
$$

for some smooth submersion $\phi: U \rightarrow \mathbb{R}$ satisfying $\operatorname{Hess}(\phi)>0$, where $\operatorname{Hess}(\phi)$ is the holomorphic Hessian:

$$
\left(\frac{\partial^{2} \phi}{\partial \bar{z}_{i} \partial z_{j}}\right)
$$

Definition 5. A strongly pseudoconvex manifold $M$ is a complex manifold with boundary which admits a strictly Levi-convex holomorphic embedding in an equidimensional complex manifold.

Definition 6. An open complex manifold $Z$ is called holomorphically convex if for every discrete closed subset $A \subset Z$ there exists a holomorphic function $Z \rightarrow \mathbb{C}$ which is proper on $A$.

Alternatively, ${ }^{2}$ one can define holomorphically convex manifolds as follows: For a compact $K$ in a complex manifold $M$, the holomorphic convex hull $\hat{K}_{M}$ of $K$ in $M$ is

$$
\hat{K}_{M}=\left\{z \in M:|f(z)| \leq \sup _{w \in K}|f(w)|, \forall f \in \mathcal{O}_{M}\right\} .
$$

In the above, $\mathcal{O}_{M}$ denotes the ring of holomorphic functions on $M$. Then $M$ is holomorphically convex iff for every compact $K \subset M$, the hull $\hat{K}_{M}$ is also compact.

\footnotetext{
${ }^{2}$ and this is the standard definition
} 
Theorem 7 (Grauert [10]). The interior of every compact strongly pseudoconvex manifold $M$ is holomorphically convex.

Definition 8. A complex manifold $M$ is called Stein if it admits a proper holomorphic embedding in $\mathbb{C}^{n}$ for some $n$.

Equivalently, $M$ is Stein iff it is holomorphically convex and holomorphically separable: That is, for every distinct points $x, y \in M$, there exists a holomorphic function $f: M \rightarrow \mathbb{C}$ such that $f(x) \neq f(y)$. We will use:

Theorem 9 (Rossi [13], Corollary on page 20). If a compact complex manifold $M$ is strongly pseudoconvex and contains no compact complex subvarieties of positive dimension, then $\operatorname{int}(M)$ is Stein.

We now discuss strong quasiconvexity and Stein property in the context of complexhyperbolic manifolds. A classical example of a complex submanifold with Levi-convex boundary is a closed round ball $\overline{\mathbf{B}}^{n}$ in $\mathbb{C}^{n}$. Suppose that $\Gamma<\operatorname{Aut}\left(\mathbf{B}^{n}\right)$ is a discrete torsionfree subgroup of the group of holomorphic automorphisms of $\mathbf{B}^{n}$ with (nonempty) domain of discontinuity $\Omega=\Omega(\Gamma) \subset \partial \mathbf{B}^{n}$. The quotient

$$
\bar{M}_{\Gamma}=\left(\mathbf{B}^{n} \cup \Omega\right) / \Gamma
$$

is a smooth manifold with boundary.

Lemma 10. $\bar{M}_{\Gamma}$ is strongly pseudoconvex.

Proof. We let $T_{\Lambda}$ denote the union of all projective hyperplanes in $P_{\mathbb{C}}^{n}$ tangent to $\partial \mathbf{B}^{n}$ at points of $\Lambda$, the limit set of $\Gamma$. Let $\widehat{\Omega}$ denote the connected component of $P_{\mathbb{C}}^{n} \backslash T_{\Lambda}$ containing $\mathbf{B}^{n}$. It is clear that $\mathbf{B}^{n} \cup \Omega \subset \widehat{\Omega}$ is strictly Levi-convex. By the construction, $\Gamma$ preserves $\widehat{\Omega}$. It is proven in $[5$, Thm. 7.5.3] that the action of $\Gamma$ on $\widehat{\Omega}$ is properly discontinuous. Hence, $X:=\widehat{\Omega} / \Gamma$ is a complex manifold containing $\bar{M}_{\Gamma}$ as a strictly Levi-convex submanifold with boundary.

Specializing to the case when $\bar{M}_{\Gamma}$ is compact, i.e. $\Gamma$ is convex-cocompact, we obtain:

Proposition 11. Suppose that $\Gamma$ is torsion-free, convex-cocompact and $n>1$. Then:

1. $\partial \bar{M}_{\Gamma}$ is connected.

2. If $\operatorname{int}\left(\bar{M}_{\Gamma}\right)=M_{\Gamma}$ contains no compact complex subvarieties of positive dimension, then $M_{\Gamma}$ is Stein.

For example, as it was observed in [4], the quotient-manifold $\mathbf{B}^{2} / \Gamma$ of a real-Fuchsian subgroup $\Gamma<\operatorname{Aut}\left(\mathbf{B}^{2}\right)$ is Stein while the quotient-manifold of a complex-Fuchsian subgroup $\Gamma<\operatorname{Aut}\left(\mathbf{B}^{2}\right)$ is non-Stein. 


\section{Proof of Theorem 1}

In this section, we construct certain plurisubharmonic functions on $M_{\Gamma}$, for each finitely generated, discrete subgroup $\Gamma<\operatorname{Aut}\left(\mathbf{B}^{n}\right)$ satisfying $\delta(\Gamma)<2$. We use these functions to show that $M_{\Gamma}$ has no compact subvarieties of positive dimension. At the end of this section, we prove the main result of this paper.

Let $X$ be a complex manifold. Recall that a continuous function $f: X \rightarrow \mathbb{R}$ is called plurisubharmonic ${ }^{3}$ if for any homomorphic map $\phi: V(\subset \mathbb{C}) \rightarrow X$, the composition $f \circ \phi$ is subharmonic. Plurisubharmonic functions $f$ satisfy the maximum principle; in particular, if $f$ restricts to a nonconstant function on a connected complex subvariety $Y \subset X$, then $Y$ is noncompact.

Now we turn to our construction of plurisubharmonic functions. Let $\Gamma<\operatorname{Aut}\left(\mathbf{B}^{n}\right)$ be a discrete subgroup. Consider the Poincaré series

$$
\sum_{\gamma \in \Gamma}\left(1-|\gamma(z)|^{2}\right), \quad z \in \mathbf{B}^{n}
$$

Lemma 12. Suppose that $\delta(\Gamma)<2$. Then (3) uniformly converges on compact sets.

Proof. Since $\delta(\Gamma)<2$, the Poincaré series

$$
\sum_{\gamma \in \Gamma} e^{-2 d(0, \gamma(z))}
$$

uniformly converges on compact subsets in $\mathbf{B}^{n}$. By (2), we get

$$
e^{-2 d(0, \gamma(z))} \leq\left(1-|\gamma(z)|^{2}\right) \leq 4 e^{-2 d(0, \gamma(z))} .
$$

Then, the result follows from the upper inequality.

Remark 13. Note that when $\delta(\Gamma)>2$, or when $\Gamma$ is of divergent type (e.g., convexcocompact) and $\delta(\Gamma)=2$, then (3) does not converge. This follows from the lower inequality of (4).

Assume that $\delta(\Gamma)<2$. Define $F: \mathbf{B}^{n} \rightarrow \mathbb{R}$,

$$
F(z)=\sum_{\gamma \in \Gamma}\left(|\gamma(z)|^{2}-1\right)
$$

Since $F$ is $\Gamma$-invariant, i.e., $F(\gamma z)=F(z)$, for all $\gamma \in \Gamma$ and all $z \in \mathbf{B}^{n}, F$ descends to a function

$$
f: M_{\Gamma} \rightarrow \mathbb{R} .
$$

\footnotetext{
${ }^{3}$ There is a more general notion of plurisubharmonic functions; for our purpose, we only consider this restrictive definition.
} 
Lemma 14. The function $f: M_{\Gamma} \rightarrow \mathbb{R}$ is plurisubharmonic.

Proof. Enumerate $\Gamma$ as $\Gamma=\left\{\gamma_{1}, \gamma_{2}, \ldots\right\}$. Consider the sequence of partial sums of the series $F$,

$$
S_{k}(z)=\sum_{j \leq k}\left(\left|\gamma_{j}(z)\right|^{2}-1\right) .
$$

Since each summand in the above is plurisubharmonic ${ }^{4}, S_{k}$ is plurisubharmonic for each $k \geq 1$. Moreover, the sequence of functions $S_{k}$ is monotonically decreasing. Thus, the limit $F=\lim _{k \rightarrow \infty} S_{k}$ is also plurisubharmonic, and hence so is $f$.

Note, however, that at this point we do not yet know that the function $f$ is nonconstant.

Now we prove the main result of this section.

Theorem 15. Let $\Gamma$ be a torsion-free discrete subgroup of $\operatorname{Aut}\left(\mathbf{B}^{n}\right)$. If $\delta(\Gamma)<2$, then $M_{\Gamma}$ contains no compact complex subvarieties of positive dimension.

Proof. Suppose that $Y$ is a compact connected subvariety of positive dimension in $M_{\Gamma}$. Since $\pi_{1}(Y)$ is finitely generated, so is its image $\Gamma^{\prime}$ in $\Gamma=\pi_{1}\left(M_{\Gamma}\right)$. Since $\delta\left(\Gamma^{\prime}\right) \leq \delta(\Gamma)$, by passing to the subgroup $\Gamma^{\prime}$ we can (and will) assume that the group $\Gamma$ is finitely generated.

We construct a sequence of functions $F_{k}: \mathbf{B}^{n} \rightarrow \mathbb{R}$ as follows. For $k \in \mathbb{N}$, let $\Sigma_{k} \subset$ $\Gamma-\{1\}$ denote the subset consisting of $\gamma \in \Gamma$ satisfying $d(0, \gamma(0)) \leq k$. Since $\Gamma$ is a finitely generated linear group, it is residually finite and, hence, there exists a finite index subgroup $\Gamma_{k}<\Gamma$ disjoint from $\Sigma_{k}$. For each $k \in \mathbb{N}$, define $F_{k}: \mathbf{B}^{n} \rightarrow \mathbb{R}$ as the sum

$$
F_{k}(z)=\sum_{\gamma \in \Gamma_{k}}\left(|\gamma(z)|^{2}-1\right)
$$

Since

$$
\bigcap_{k \in \mathbb{N}} \Gamma_{k}=\{1\}
$$

the sequence of functions $F_{k}$ converges to $\left(|z|^{2}-1\right)$ uniformly on compact subsets of $\mathbf{B}^{n}$. As before, each $F_{k}$ is plurisubharmonic (cf. Lemmata 12, 14).

Let $\widetilde{Y}$ be a connected component of the preimage of $Y$ under the projection map $\mathbf{B}^{n} \rightarrow M_{\Gamma}$. Since $\widetilde{Y}$ is a closed, noncompact subset of $\mathbf{B}^{n}$, the function $\left(|z|^{2}-1\right)$ is nonconstant on $\tilde{Y}$. As the sequence $\left(F_{k}\right)$ converges to $\left(|z|^{2}-1\right)$ uniformly on compacts, there exists $k \in \mathbb{N}$ such that $F_{k}$ is nonconstant on $\widetilde{Y}$. Let $f_{k}: M_{k}=M_{\Gamma_{k}} \rightarrow \mathbb{R}$ denote the function obtained by projecting $F_{k}$ to $M_{k}$, and $Y_{k}$ be the image of $\tilde{Y}$ under the projection map $\mathbf{B}^{n} \rightarrow M_{k}$. Since $M_{k}$ is a finite covering of $M_{\Gamma}$, the subvariety $Y_{k} \subset M_{k}$ is compact. Moreover, $f_{k}$ is a nonconstant plurisubharmonic function on $Y_{k}$ since $F_{k}$ is such a function on $\widetilde{Y}$. This contradicts the maximum principle.

\footnotetext{
${ }^{4}$ This follows from the fact that the function $|z|^{2}$ is plurisubharmonic.
} 
Remark 16. Regarding Remark 13: The failure of convergence of the series (3) as pointed out in Remark 13 is not so surprising. In fact, if $\Gamma$ is a complex Fuchsian group, then $\delta(\Gamma)=2$ and the convex core of $M_{\Gamma}$ is a compact Riemann surface, see Example 4. Thus, our construction of $F$ must fail in this case.

We conclude this section with a proof of the main result of this paper.

Proof of Theorem 1. By Theorem 15, $M_{\Gamma}$ does not have compact complex subvarieties of positive dimensions. Then, by the second part of Proposition 11, $M_{\Gamma}$ is Stein.

\section{Further remarks}

In relation to Theorem 1, it is also interesting to understand the case when $\delta(\Gamma)=2$, that is: For which convex-cocompact, torsion-free subgroups $\Gamma$ of $\operatorname{Aut}\left(\mathbf{B}^{n}\right)$ satisfying $\delta(\Gamma)=2$, is the manifold $M_{\Gamma}$ Stein? It has been pointed out before that a complex Fuchsian subgroup $\Gamma<\operatorname{Aut}\left(\mathbf{B}^{n}\right)$ satisfies $\delta(\Gamma)=2$, but the manifold $M_{\Gamma}$ is not Stein. In fact, the convex core of $M_{\Gamma}$ is a complex curve, see Remark 16. We conjecture that complex Fuchsian subgroups are the only such non-Stein examples.

Conjecture 17. Let $\Gamma<\operatorname{Aut}\left(\mathbf{B}^{n}\right)$ be a convex-cocompact, torsion-free subgroup such that $\delta(\Gamma)=2$. Then, $M_{\Gamma}$ is non-Stein if and only if $\Gamma$ is a complex Fuchsian subgroup.

We illustrate this conjecture in the following very special case: Let $\phi: \pi_{1}(\Sigma) \rightarrow \operatorname{Aut}\left(\mathbf{B}^{n}\right)$ be a faithful convex-cocompact representation where $\Sigma$ is a compact Riemann surface of genus $g \geq 2$. Then $\phi$ induces a (unique) equivariant harmonic map

$$
F: \widetilde{\Sigma} \rightarrow \mathbf{B}^{n} .
$$

which descends to a harmonic map $f: \Sigma \rightarrow M_{\Gamma}$.

Proposition 18. Suppose that $F$ is a holomorphic immersion. Then $\Gamma=\phi\left(\pi_{1}(\Sigma)\right)$ satisfies $\delta(\Gamma) \geq 2$. Moreover, if $\delta(\Gamma)=2$, then $\Gamma$ preserves a complex line. In particular, $\Gamma$ is a complex Fuchsian subgroup of $\operatorname{Aut}\left(\mathbf{B}^{n}\right)$.

Proof. Noting that $M_{\Gamma}$ contains a compact complex curve, namely $f(\Sigma)$, the first part follows directly from Theorem 1.

For the second part, we let $Y$ denote the surface $\tilde{\Sigma}$ equipped with the Riemannian metric obtained via pull-back of the Riemannian metric $g$ on $\mathbf{B}^{n}$. The entropy ${ }^{5} h(Y)$ of $Y$ is bounded above by $\delta(\Gamma)$, i.e.

$$
h(Y) \leq 2 .
$$

\footnotetext{
${ }^{5}$ The volume entropy of a simply connected Riemannian manifold $(X, g)$ is defined as $\lim _{r \rightarrow \infty} \log \operatorname{Vol}(B(r, x)) / r$, where $x \in X$ is a chosen base-point and $B(r, x)$ denotes the ball of radius $r$ centered at $x$. This limit exists and is independent of $x$, see [12].
} 
This can be seen as follows: The distance function $d_{Y}$ on $Y$ satisfies

$$
d_{Y}\left(y_{1}, y_{2}\right) \geq d\left(F\left(y_{1}\right), F\left(y_{2}\right)\right) .
$$

Therefore, the exponential growth-rate $\delta_{Y}$ of $\pi_{1}(\Sigma)$-orbits in $Y$ satisfies $\delta_{Y} \leq \delta(\Gamma)$. On the other hand, the quantity $\delta_{Y}=h(Y)$ since $\pi_{1}(\Sigma)$ acts cocompactly on $Y$.

Assume that $\widetilde{\Sigma}$ is endowed with a conformal Riemannian metric of constant -4 sectional curvature. Since $\tilde{\Sigma}$ is a symmetric space, we have

$$
h^{2}(Y) \operatorname{Area}(Y / \Gamma) \geq h^{2}(\widetilde{\Sigma}) \operatorname{Area}(\Sigma)
$$

see $[1$, p. 624]. The inequality (5) together with the above implies that $\operatorname{Area}(Y / \Gamma) \geq$ $\operatorname{Area}(\Sigma)$.

On the other hand, since $f: Y / \Gamma \rightarrow M_{\Gamma}$ is holomorphic, $4 \cdot$ Area $(Y / \Gamma)$ equals to the Toledo invariant $c(\phi)$ (see [14]) of the representation $\phi$. Since $c(\phi) \leq 4 \pi(g-1)$, the inequality $\operatorname{Area}(Y / \Gamma) \geq \operatorname{Area}(\Sigma)=\pi(g-1)$ shows that $\operatorname{Area}(Y / \Gamma)=\pi(g-1)$ or, equivalently, $c(\phi)=4 \pi(g-1)$. By the main result of [14], $\Gamma$ preserves a complex-hyperbolic line in $\mathbf{B}^{n}$.

Remark 19. The assumption that $F$ is an immersion can be eliminated: Instead of working with a Riemannian metric, one can work with a Riemannian metric with finitely many singularities.

Motivated by Theorem 15, we also make the following conjecture.

Conjecture 20. If $\Gamma<\operatorname{Aut}\left(\mathbf{B}^{n}\right)$ is discrete, torsion-free, and $\delta(\Gamma)<2 k$, then $M_{\Gamma}$ does not contain compact complex subvarieties of dimension $\geq k$.

We conclude this section with a verification of this conjecture under a stronger hypothesis.

Proposition 21. If $\Gamma<\operatorname{Aut}\left(\mathbf{B}^{n}\right)$ is discrete, torsion-free, and $\delta(\Gamma)<2 k-1$, then $M_{\Gamma}$ does not contain compact complex subvarieties of dimension $\geq k$.

Proof. Note that if $\Gamma$ is elementary (i.e., virtually abelian), then $\delta(\Gamma)=0$. In this case, the result follows from Theorem 15. For the rest, we assume that $\Gamma$ is nonelementary.

By $\left[2\right.$, Sec. 4], there is a natural map $f: M_{\Gamma} \rightarrow M_{\Gamma}$ homotopic to the identity map $\operatorname{id}_{M_{\Gamma}}: M_{\Gamma} \rightarrow M_{\Gamma}$ and satisfying

$$
\left|\operatorname{Jac}_{p}(f)\right| \leq\left(\frac{\delta(\Gamma)+1}{p}\right)^{p}, \quad 2 \leq p \leq 2 n
$$

where $\operatorname{Jac}_{p}(f)$ denotes the $p$-Jacobian of $f$. When $\delta(\Gamma)<2 k-1$, we have $\left|\operatorname{Jac}_{p}(f)\right|<1$, for $p \in[2 k, 2 n]$. This means that $f$ strictly contracts the volume form on each $p$-dimensional tangent space at every point $x \in M_{\Gamma}$, for $p \in[2 k, 2 n]$. 
Let $Y \subset M_{\Gamma}$ be a compact complex subvariety of dimension $\geq k$ (real dimension $\geq 2 k$ ). Then, $Y$ is also a volume minimizer in its homology class. Since $f$ strictly contracts volume on $Y, f(Y)$ has volume strictly lesser than that of $Y$. However, $f$ being homotopic to $\operatorname{id}_{M_{\Gamma}}, f(Y)$ belongs to the homology class of $Y$. This is a contradiction to the fact that $Y$ minimizes volume its homology class.

Remark 22. Note that Proposition 21 gives an alternative proof of Theorem 15 (hence Theorem 1) under a stronger hypothesis, namely $\delta(\Gamma) \in(0,1)$. However, this method fails to verify Theorem 15 in the case when $\delta(\Gamma) \in[1,2)$.

Finally, we note that the papers [6] and [16] contain other interesting results and conjectures on Stein properties of complex-hyperbolic manifolds.

\section{References}

[1] G. Besson, G. Courtois, and S. Gallot. Minimal entropy and Mostow's rigidity theorems. Ergodic Theory Dynam. Systems, 16(4):623-649, 1996.

[2] G. Besson, G. Courtois, and S. Gallot. Rigidity of amalgamated products in negative curvature. J. Differential Geom., 79(3):335-387, 2008.

[3] B. H. Bowditch. Geometrical finiteness with variable negative curvature. Duke Math. J., 77(1):229-274, 1995.

[4] D. Burns, Jr. and S. Shnider. Spherical hypersurfaces in complex manifolds. Invent. Math., 33(3):223-246, 1976.

[5] A. Cano, J. P. Navarrete, and J. Seade. Complex Kleinian groups, volume 303 of Progress in Mathematics. Birkhäuser/Springer Basel AG, Basel, 2013.

[6] B.-Y. Chen. Discrete groups and holomorphic functions. Math. Ann., 355(3):10251047, 2013.

[7] K. Corlette. Hausdorff dimensions of limit sets. I. Invent. Math., 102(3):521-541, 1990.

[8] K. Corlette and A. Iozzi. Limit sets of discrete groups of isometries of exotic hyperbolic spaces. Trans. Amer. Math. Soc., 351(4):1507-1530, 1999.

[9] W. M. Goldman. Complex hyperbolic geometry. Oxford Mathematical Monographs. The Clarendon Press, Oxford University Press, New York, 1999. Oxford Science Publications.

[10] H. Grauert. On Levi's problem and the imbedding of real-analytic manifolds. Ann. of Math. (2), 68:460-472, 1958. 
[11] M. Kapovich. Lectures on complex hyperbolic Kleinian groups. arxiv:1911.12806, 2019.

[12] A. Manning. Topological entropy for geodesic flows. Ann. of Math. (2), 110(3):567$573,1979$.

[13] H. Rossi. Strongly pseudoconvex manifolds. In Lectures in Modern Analysis and Applications, I, pages 10-29. Springer, Berlin, 1969.

[14] D. Toledo. Representations of surface groups in complex hyperbolic space. J. Differential Geom., 29(1):125-133, 1989.

[15] A. Weil. Remarks on the cohomology of groups. Ann. of Math. (2), 80:149-157, 1964.

[16] C. Yue. Webster curvature and Hausdorff dimension of complex hyperbolic Kleinian groups. In: Y. Jiang and L. Wen, editors, Dynamical Systems-Proceedings Of The International Conference In Honor Of Professor Liao Shantao, pages 319-328. World Sci. Publ., Singapore, 1999. 